PROCEEDINGS OF THE

AMERICAN MATHEMATICAL SOCIETY

Volume 136, Number 7, July 2008, Pages 2565-2570

S 0002-9939(08)09484-7

Article electronically published on March 14, 2008

\title{
ON DECAY OF SOLUTIONS TO NONLINEAR SCHRÖDINGER EQUATIONS
}

\author{
ALEXANDER PANKOV
}

(Communicated by Michael Weinstein)

\begin{abstract}
We present general results on exponential decay of finite energy solutions to stationary nonlinear Schrödinger equations. Under certain natural assumptions we show that any such solution is continuous and vanishes at infinity. This allows us to interpret the solution as a finite multiplicity eigenfunction of a certain linear Schrödinger operator and, hence, apply well-known results on the decay of eigenfunctions.
\end{abstract}

In this note we consider the equation

$$
-\Delta u+V(x) u=f(x, u), \quad x \in \mathbb{R}^{n},
$$

and, under rather general assumptions, derive exponential decay estimates for its solutions.

We suppose that

(i) The potential $V$ belongs to $L_{\mathrm{loc}}^{\infty}\left(\mathbb{R}^{n}\right)$ and is bounded below, i.e. $V(x) \geq-c_{0}$ for some $c_{0} \in \mathbb{R}$.

Under assumption $(i)$ the left hand side of equation (11) defines a self-adjoint operator in $L^{2}\left(\mathbb{R}^{n}\right)$ denoted by $H$. The operator $H$ is bounded below. We suppose that

(ii) The essential spectrum $\sigma_{\mathrm{ess}}(H)$ of the operator $H$ does not contain the point 0 .

Note, however, that 0 can be an eigenvalue of finite multiplicity.

The nonlinearity of $f$ is supposed to satisfy the following assumption.

(iii) The function $f(x, u)$ is a Carathéodory function; i.e. it is Lebesgue measurable with respect to $x \in \mathbb{R}^{n}$ for all $u \in \mathbb{R}$ and continuous with respect to $u \in \mathbb{R}$ for almost all $x \in \mathbb{R}^{n}$. Furthermore,

$$
|f(x, u)| \leq c\left(1+|u|^{p-1}\right), \quad x \in \mathbb{R}^{n} u \in \mathbb{R},
$$

with $c>0$ and $2 \leq p<2^{*}$, where

$$
2^{*}= \begin{cases}\frac{2 n}{n-2} & \text { if } n \geq 3, \\ \infty & \text { if } n=1,2,\end{cases}
$$

Received by the editors September 18, 2006, and, in revised form, June 29, 2007.

2000 Mathematics Subject Classification. Primary 35J60, 35B40.

Key words and phrases. Nonlinear Schrödinger equation, exponential decay.

(C)2008 American Mathematical Society Reverts to public domain 28 years from publication 
and

$$
\lim _{u \rightarrow 0} \operatorname{ess}_{\sup } \frac{|f(x, u)|}{|u|}=0 .
$$

Let $E$ denote the form domain of the operator $H$, i.e., the domain of the corresponding quadratic form or, which is the same, the domain of the operator $H^{1 / 2}$. It is well-known that

$$
E=\left\{u \in H^{1}\left(\mathbb{R}^{n}\right):\left(V(x)+c_{0}+1\right) u(x) \in L^{2}\left(\mathbb{R}^{n}\right)\right\}
$$

where $c_{0}$ is the constant from assumption $(i)$. Moreover, $E$ carries a natural Hilbert space structure and is continuously embedded into the space $H^{1}\left(\mathbb{R}^{n}\right)$. In what follows we consider only weak solutions that belong to the space $E$. A function $u \in E$ is a weak solution of equation (11) if for all $v \in E$ the integral identity

$$
\int_{\mathbb{R}^{n}}(\nabla u(x) \cdot \nabla v(x)+V(x) u(x) v(x)-f(x, u(x)) v(x)) d x=0
$$

is satisfied. Actually, it is sufficient to check identity (3) only for functions $v$ that belong to the space $C_{0}^{\infty}\left(\mathbb{R}^{n}\right)$ of all finitely supported infinitely differentiable functions. Also we note that, due to the Sobolev embedding theorem, the term $f(x, u(x)) v(x)$ in (3) is integrable for all $u, v \in H^{1}\left(\mathbb{R}^{n}\right)$.

The existence of (nontrivial) finite energy solutions is one of the main problems concerning equation (11). There are many papers in this direction, but we do not address the existence problem here. We only mention that in most such results assumptions (i)-(iii) are satisfied. For instance, let us consider the equation

$$
-\Delta u+V(x) u=|u|^{p-2} u, \quad x \in \mathbb{R}^{n},
$$

with $2<p<2^{*}$. Equation (4) possesses a nontrivial finite energy solution at least in the following two cases: $(a)$ the potential $V$ is periodic and 0 is in a spectral gap of the operator $H$ [8, 10, 16, (b) $V(x) \rightarrow+\infty$ as $x \rightarrow \infty$ [12. In case $(b)$ the spectrum of the operator $H$ is discrete. An introductory exposition and references concerning the existence problem can be found in [11.

There are a number of results on exponential decay of solutions to equation (1) (see 11, 3, 4, 10, 11, 15]). Most of them, except [10, 11, deal with the case when 0 is below the essential spectrum of $H$. However, the case when 0 is in a spectral gap is extremely important for applications [10]. Here we present a rather general result in this direction.

We exploit the following rather simple idea. Suppose that $u \in E$ is a nontrivial, i.e. $u \neq 0$, solution to equation (11). Set

$$
W(x)= \begin{cases}\frac{f(x, u(x))}{u(x)} & \text { if } u(x) \neq 0, \\ 0 & \text { if } u(x)=0 .\end{cases}
$$

Then equation (11) can be represented as

$$
(H+W) u=0 .
$$

This means that $u$ is an eigenfunction of the operator $H+W$ with zero eigenvalue. Now if the multiplication operator by $W$ is relatively compact with respect to the operator $H$, then

$$
\sigma_{\mathrm{ess}}(H+W)=\sigma_{\mathrm{ess}}(H)
$$


Hence, 0 is an eigenvalue of $H+W$ of finite multiplicity and $u$ a corresponding eigenfunction (see, e.g., 6, 13]). Now an exponential decay of $u$ can be read from any well-known result about eigenfunctions. More precisely, the solution $u$ has exactly the same decay as an eigenfunction that corresponds to an eigenvalue introduced into a spectral gap by a decaying perturbation of the potential $V$.

Thus, we only need to verify that the multiplication operator by the function $W$ is relatively compact with respect to $H$. The key point is the following:

Lemma 1. Under assumptions ( $i)-($ iii) suppose that $u \in E$ is a solution of equation (11). Then $u$ is a continuous function and

$$
\lim _{x \rightarrow \infty} u(x)=0 .
$$

We postpone the proof of the lemma and first present the main results.

Due to a well-known result (see, e.g., Theorem 8.3.1 of [11), Lemma 1 implies that the multiplication operator by $W$ is a relatively compact perturbation of the operator H. Making use of Theorem C.3.4, 14, we obtain

Theorem 2. Assume (i)-(iii). Let $u \in E$ be a solution of equation (11). Then there exists $\alpha_{0}>0$ such that for every $\alpha<\alpha_{0}$ we have

$$
|u(x)| \leq C \exp (-\alpha|x|)
$$

with some $C=C_{\alpha}>0$.

Remark 3. An interesting case is when the potential is periodic. If, in addition, the nonlinearity is superlinear, i.e. $f(\cdot, u) \geq c|u|^{p-1}$ at infinity, with $p>2$, the result of Theorem 2 is announced in [10]. However, assumption (iii) allows asymptotically linear nonlinearities $(p=2)$. As a consequence, solutions found in [9] decay exponentially fast.

Remark 4. The value of $\alpha_{0}$ can be estimated in terms of the distance between 0 and $\sigma_{\text {ess }}(H)$ (see, e.g., [5]).

Now we consider the case when $\sigma_{\text {ess }}(H)=\varnothing$; i.e. the spectrum of $H$ is discrete. This is so if, e.g.,

$$
\lim _{|x| \rightarrow \infty} V(x)=\infty
$$

For a necessary and sufficient condition for the discreteness of spectrum, see [7] and the references therein. In this case Theorem C.3.3 of [14] implies

Theorem 5. Under assumptions (i)-(iii), suppose that the spectrum of $H$ is discrete. Let $u \in E$ be a solution of equation (11). Then for every $\alpha>0$ there exists $C=C_{\alpha}>0$ such that

$$
|u(x)| \leq C \exp (-\alpha|x|) .
$$

Having additional information about the behavior of $V$ at infinity, one can refine the result of Theorem [5. For instance, making use of Theorem 3.3 of [2], we obtain

Theorem 6. In addition to assumptions (i)-(iii), suppose that

$$
V(x) \geq \gamma|x|^{\beta}-\gamma_{0}
$$

with $\gamma>0, \gamma_{0} \geq 0$ and $\beta>0$. Then for any solution $u \in E$ of equation (1) we have that

$$
\mid u(x) \leq C \exp \left(-a|x|^{\frac{\beta}{2}+1}\right),
$$

with some $C>0$ and $a>0$. 
We now prove Lemma 1 .

Proof of Lemma 1. The case $n=1$ is trivial because any function from $H^{1}(\mathbb{R})$ is continuous and vanishes at infinity.

Now we consider the case $n \geq 3$. We use a version of the well-known bootstrap argument as follows.

Equation (11) can be rewritten as

$$
\left(-\Delta+V+c_{0}+1\right) u=\left(c_{0}+1\right) u+f(x, u) .
$$

Note that by the Sobolev embedding, $u \in L^{2^{*}}\left(\mathbb{R}^{n}\right)$.

Suppose now that $u \in L^{\infty}\left(\mathbb{R}^{n}\right)+L^{r}\left(\mathbb{R}^{n}\right)$ with $r \geq 2^{*}$. Let

$$
A=\left\{x \in \mathbb{R}^{n}:|u(x)| \geq 1\right\}
$$

and $B=\mathbb{R}^{n} \backslash A$. It is easy to see that meas $(A)<\infty$. Denote by $\chi_{A}$ and $\chi_{B}$ the characteristic functions of the sets $A$ and $B$, respectively, i.e. $\chi_{A}=1$ on $A, \chi_{A}=0$ on $B$ and $\chi_{B}=1-\chi_{A}$.

Let

$$
\begin{gathered}
H_{1}=-\Delta+V(x)+c_{0}+1, \\
h_{0}(x)=\chi_{B}(x)\left[\left(c_{0}+1\right) u(x)+f(x, u)\right],
\end{gathered}
$$

and

$$
h_{1}(x)=\chi_{A}(x)\left[\left(c_{0}+1\right) u(x)+f(x, u)\right] .
$$

Equation (10) becomes

$$
H_{1} u=h_{0}(x)+h_{1}(x)
$$

Obviously, $h_{0} \in L^{\infty}\left(\mathbb{R}^{n}\right)$, while assumption (iii) implies that $h_{1} \in L^{s}\left(\mathbb{R}^{n}\right)$ where

$$
s=\frac{r}{p-1} .
$$

The operator $H_{1}$ is positive definite and satisfies the assumptions of the Sobolev estimate theorem for Schrödinger operators (see Theorem B.2.1 of [14]). Hence, we obtain from (11) that $u=u_{0}+u_{1}$, where

$$
u_{0}=H_{1}^{-1} h_{0} \in L^{\infty}\left(\mathbb{R}^{n}\right)
$$

and

for every $q$ such that

$$
u_{1}=H_{1}^{-1} h_{1} \in L^{q}\left(\mathbb{R}^{n}\right)
$$

$$
\frac{1}{s}-\frac{1}{q}<\frac{2}{n}
$$

If $s>n / 2$, i.e.

$$
r>\frac{n(p-1)}{2},
$$

we can take $q=\infty$ to obtain $u_{1} \in L^{\infty}\left(\mathbb{R}^{n}\right)$ and, hence, $u=u_{0}+u_{1} \in L^{\infty}\left(\mathbb{R}^{n}\right)$.

Otherwise, take

where

$$
q=\frac{r}{1-\delta},
$$

$$
\delta=\frac{4}{n-2}-(p-2)-\varepsilon
$$


and $\varepsilon>0$ is arbitrarily small. Note that

$$
p-2<\frac{4}{n-2}
$$

because $p<2^{*}$. Since $r \geq 2^{*}$, we have that

$$
\begin{aligned}
\frac{1}{s}-\frac{1}{q} & =\frac{p-1}{r}-\frac{1-\delta}{r}=\frac{1}{r}\left(\frac{4}{n-2}-\varepsilon\right) \leq \frac{1}{2^{*}}\left(\frac{4}{n-2}-\varepsilon\right) \\
& =\frac{2}{n}-\frac{n-2}{2 n} \varepsilon .
\end{aligned}
$$

Therefore, $q$ satisfies (12) and $u_{1} \in L^{q}\left(\mathbb{R}^{n}\right)$. Hence,

$$
u=u_{0}+u_{1} \in L^{\infty}\left(\mathbb{R}^{n}\right)+L^{q}\left(\mathbb{R}^{n}\right) .
$$

It is not difficult to verify that $\chi_{A} u \in L^{q}\left(\mathbb{R}^{n}\right)$ and $h_{1} \in L^{q /(p-1)}\left(\mathbb{R}^{n}\right)$.

Now starting with $r=r_{0}=2^{*}$, we can iterate the previous procedure. Thus, we have that $u \in L^{\infty}\left(\mathbb{R}^{n}\right)+L^{r_{k}}\left(\mathbb{R}^{n}\right)$, where

$$
r_{k}=\frac{2^{*}}{(1-\delta)^{k}}
$$

Let $k$ be so large that

$$
r_{k}>\frac{n(p-1)}{2}
$$

(see (13) $)$. Then

$$
s_{k}=\frac{r_{k}}{p-1}>\frac{n}{2}
$$

and we can apply the Sobolev estimate of Theorem B.2.1 of 14 with $s=s_{k}$ and $q=\infty$. Therefore, $u_{1} \in L^{\infty}\left(\mathbb{R}^{n}\right)$ and, hence, $u=u_{0}+u_{1} \in L^{\infty}\left(\mathbb{R}^{n}\right)$.

This immediately implies that the additional potential $W$ belongs to $L_{\text {loc }}^{\infty}\left(\mathbb{R}^{n}\right)$ and is bounded below. Therefore, due to Theorems C.1.1 and C.3.1 of [14 the result follows.

The case $n=2$ is simpler. By the Sobolev embedding, $u \in L^{r}\left(\mathbb{R}^{n}\right)$ for arbitrarily large $r$. Hence, the previous argument shows that $u \in L^{\infty}\left(\mathbb{R}^{n}\right)$, and we are done.

\section{REFERENCES}

1. T. Bartsch, A. Pankov, Zh.-Q. Wang, Nonlinear Schrödinger equation with a steep potential well, Commun. Contemp. Math. 3 (2001), 549-569. MR1869104(2002k:35079)

2. F. A. Berezin, M. A. Shubin, The Schrödinger Equation, Kluwer, Dordrecht, 1991. MR.1186643 (93i:81001)

3. H. Berestycki, P.-L. Lions, Nonlinear scalar field equations, I. Existence of a ground state, Arch. Rat. Mech. Anal. 82 (1983), 313-345. MR695535 (84h:35054a)

4. R. Fukuizumi, T. Ozawa, Exponential decay of solutions to nonlinear elliptic equations with potentials, Z. Angew. Math. Phys. 56 (2005), 1000-1011. MR2187002 (2006i:35089)

5. P. D. Hislop, Exponential decay of two-body eigenfunctions: A review, Math. Phys. Quant. Field Theory, Electr. J. Differ. Equat. Conf. 4, 2000, pp. 265-288. MR.1785381 (2001j:81247)

6. P. D. Hislop, I. M. Sigal, Introduction to Spectral Theory, with Applications to Schrödinger Operators, Springer-Verlag, New York, 1996. MR1361167 (98h:47003)

7. V. Kondrat'ev, M. Shubin, Discreteness of spectrum for the Schrödinger operators on manifolds of bounded geometry, Oper. Theory Adv. Appl., Vol. 110, Birkhäuser, Basel, 1999, pp. 185-226. MR 1747895 (2001c:58030)

8. W. Kryszewski, A. Szulkin, Generalized linking theorem with an application to a semilinear Schrödinger equation, Adv. Differ. Equat. 3 (1998), 441-472. MR1751952 (2001g:58021) 
9. G. Li, A. Szulkin, An asymptotically periodic Schrödinger equation with indefinite linear part, Commun. Contemp. Math. 4 (2002), 763-776. MR.1938493 (2004b:35104)

10. A. Pankov, Periodic nonlinear Schrödinger equation with application to photonic crystals, Milan J. Math. 73 (2005), 259-287. MR2175045 (2006h:35087)

11. A. Pankov, Lecture Notes on Schrödinger Equations, Nova Publ., 2007.

12. P. H. Rabinowitz, On a class of nonlinear Schrödinger equations, Z. Angew. Math. Phys. 43 (1992), 270-291. MR1162728 (93h:35194)

13. M. Reed, B. Simon, Methods of Modern Mathematical Physics, IV: Analysis of Operators, Academic Press, New York, 1978. MR0493421 (58:12429c)

14. B. Simon, Schrödinger semigroups, Bull. Amer. Math. Soc. (N.S.) 7 (1982), 447-526. MR670130 (86b:81001a)

15. C. A. Stuart, An introduction to elliptic equations on $\mathbb{R}^{n}$, Nonlinear Functional Analysis and Applications to Differential Equations, Trieste, 1997, World Sci. Publ., River Edge, NJ, 1998, pp. 237-285. MR 1703533 (2000f:35025)

16. M. Willem, Minimax Theorems, Birkhäuser, Boston, 1996. MR1400007 (97h:58037)

Department of Mathematics, College of William and Mary, Williamsburg, Virginia $23187-8795$

Current address: Department of Mathematics, Morgan State University, Baltimore, Maryland 21251

E-mail address: pankov@member.ams.org 